Dear author,

Please note that changes made in the online proofing system will be added to the article before publication but are not reflected in this PDF.

We also ask that this file not be used for submitting corrections.

This is the final peer-reviewed accepted manuscript of:

CarloMengucci, AlessandraBordoni, FrancescoCapozzi,

Understanding the Kinetics of Nutrients Bioaccessibility

by Modelling Foodomics Data

https://doi.org/10.1016/j.cofs.2020.04.001 


\section{Understanding the kinetics of nutrients bioaccessibility} by modelling foodomics data

${ }_{301}$ Carlo Mengucci ${ }^{1}$, Alessandra Bordoni ${ }^{1,2}$ and

${ }_{4}$ Francesco Capozzi ${ }^{1,2}$

5 Holistic methods at the basis of the foodomics approach are allowing the in-depth understanding, at molecular and supramolecular level, of the complexity of food matrix. The latter, in turn, affects the nutrient bioaccessibility, one of the crucial factors impacting on the final effect of diets. However, many levels of complexity are emerging, relating to foodhuman interactions, while bolus descends along the whole gastrointestinal tract. Such complexity makes in-vitro and insilico models still unable to fully describe intertwined kinetics between food matrix and human compartments. A possible framework to unravel complexity is outlined, starting from bioaccessibility modelling all the way down to intercompartmental kinetics. The aim is to enhance algorithms and models for the prediction of the impact of a food category on a class of individuals. The proposed framework can consider many levels of complexity, provided that time-resolved experiments, suitable for integration with food matrix description, are correctly designed for this purpose.

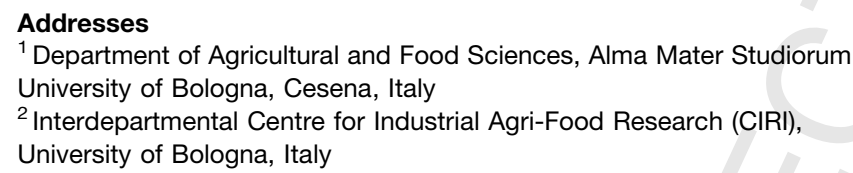

most important, other, equally important, variables emerge. Among them, the food structure and the interaction between food and the human gastrointestinal tract (GIT) are fundamental. Therefore, a 'precision nutrition' approach should consider not only individual variability (i.e. genetics, type of microbiome, metabolome, dietary habits, lifestyle) [2] but also food structure and composition, along with dynamics of digestion and absorption.

At present, the evaluation of nutrient intake is mainly based on chemical composition of consumed food and does not consider bioaccessibility, that is, the amount of the food components that is released from the food matrix, and bioavailability, that is, the amount of bioaccessible components that is absorbed and delivered to tissues through the blood stream. Since the food matrix and processing have a significant impact on bioaccessibility, which in turn impacts on bioavailability, a holistic approach to food characterization is needed. The foodomics approach offers not only a high-resolution food description, dealing with the various levels of complexity converging into food science [3], but also the in-depth description of the food metabolome.

The food metabolome is the part of the human metabolome directly derived from the digestion, absorption and biotransformation of foods and their constituents [4]. Thus, the food metabolome strictly depends on bioaccessibility and bioavailability kinetics. Nuclear Magnetic Resonance (NMR) spectroscopy and Mass Spectrometry (MS), hyphenated or not to chromatographic separation methods, are optimal techniques to comprehensively characterize the food metabolome, which can be considered one of the dimensions of the foodomics space [5]. The different levels of information in the food metabolome can be explored by i) targeted metabolite analysis, ii) metabolite profiling, iii) spectral fingerprinting, iv) untargeted metabolite analysis and v) metabolomics, with increasing discrimination capability.

To fully understand the food metabolome, the behaviour of food and food components along the gastrointestinal tract (GIT) must be considered (Figure 1). In-vitro and insilico models have been developed to simulate digestion and absorption, allowing to build up predictive models $[6,7]$. Predictive models need validation using blood, urine and faeces obtained from carefully designed intervention trials, including data quality control protocols.
41

42 43 44 45 46 47 48 


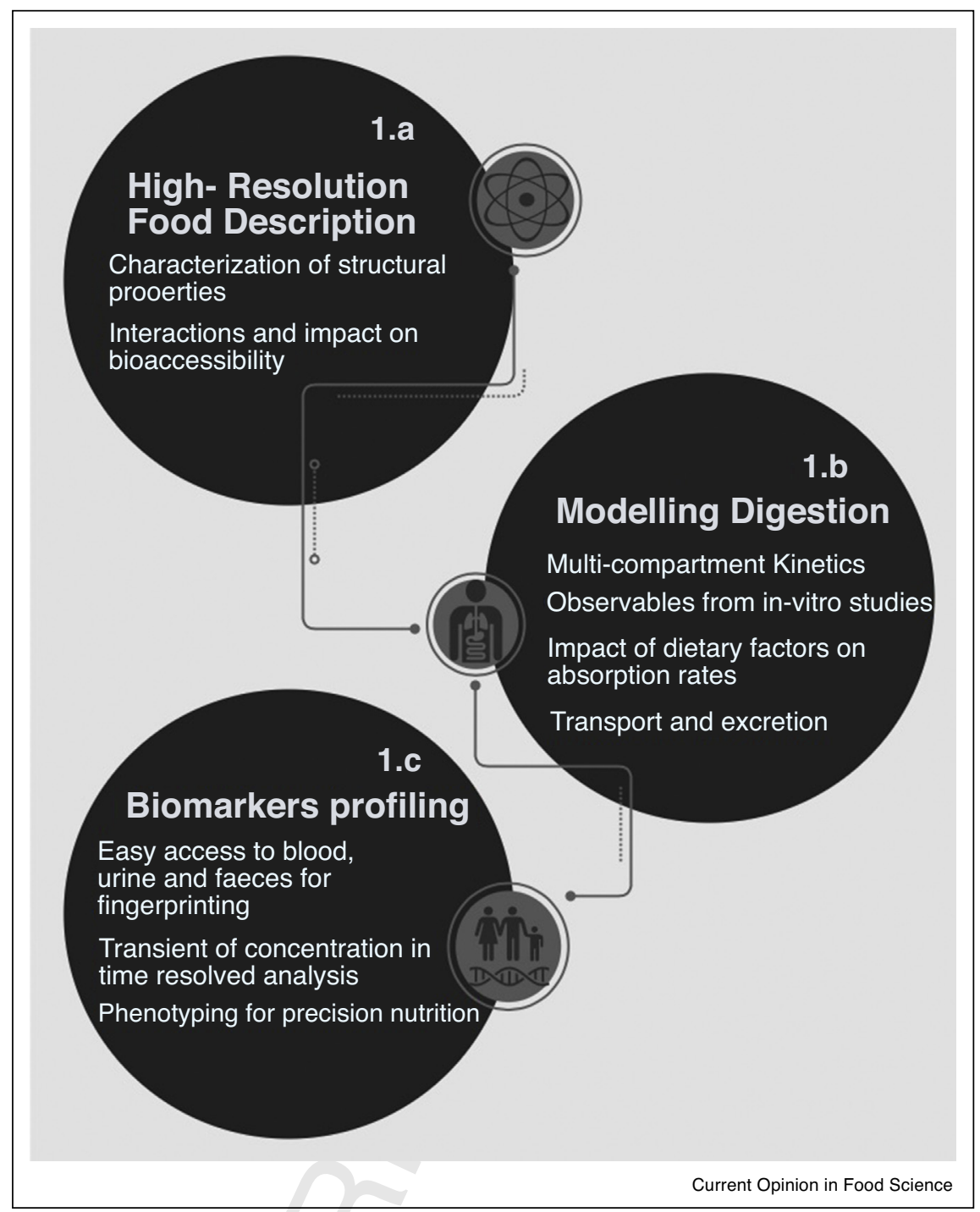

Framework for kinetics of bioavailability investigation.

The three main stages of modelling foodomics data are highlighted: 1a) numerical descriptors for the food matrix are required to be included as input in the machine learning system: 1b) modelling will find the right parameters for matching the food intake to the experimental time-resolved concentrations of food biomarkers; 1c) the set of output parameters, extracted upon modelling of food-biomarkers kinetics in blood and urine, are condensed signatures of metabolic phenotypes, linked to nutritional response to specific food products.

Samples from well-designed intervention trials can also be used to select specific biomarkers of intake. These biomarkers reflect the interactions between the food and the human body and can be used to build up in-silico models to predict bioaccessibility and bioavailability, thus allowing the classification of foods, diets and human subjects. To this purpose, the kinetic constants that regulate mass transfers between the different body compartments (including GTI) are crucial. Therefore, to develop accurate in-silico kinetic models, the time-dependent concentrations of biomarkers in different body compartments must be assessed.
Within the framework of the FoodBAll project [8], many databases have been developed (FooDB, Exposome Explorer) merging data obtained from samples coming from intervention studies. One of the main concerns emerged in the project is the transient concentration of food-related molecules, which makes their classification as biomarkers extremely difficult. Indeed, a food-related molecule may not be recognized as biomarker of intake depending on its absorption kinetics. In fact, its concentration at the time of sampling may not be different from baseline because it has not reached the peak yet (subjects with slow absorption kinetics) or it has already passed the 
peak (subjects with fast kinetics). The use of modelling can overcome this limitation, and it can also consider the 'food matrix effect'.

Although recent works highlight the importance of developing personalized wellness tools relying on data integration and biomarker mapping approaches $\left[9,10^{\bullet \bullet}\right]$, a consensus solution is far from being accepted since the derived in-silico models are not yet validated and are still at an embryonic stage.

In this paper, we discuss the possible integration of invitro experiments (data sources) with machine learning approaches aiming at extracting molecular features (datadriven approaches) to give rise to in-silico modelling able to predict kinetics of biomarkers in different compartments. We outline a framework for merging different levels of complexity by discussing methodologies and challenges for food-human interactions while stressing the importance of: i) choosing proper in-vitro descriptors for the food matrix; ii) identifying in-vivo biomarkers of food intake within pattern clustering and fingerprinting techniques; iii) integrating food matrix descriptors with biomarkers kinetics.

\section{Discussion}

The work by Westerman $e t a l$. $\left[10^{\bullet \bullet}\right]$ outlined a promising direction for nutritional recommendations based on custom biomarker correlation mapping. In that work, a set of common blood biomarkers of health was organized in a network of correlations, whose variations were studied over time. This approach allowed finding new patterns or 'networks of predictive biomarkers' to better understand transitions between health and disease states. Such patterns resulted in valuable information about the average baseline functional complexity and a subject-dependent variability. New correlations between biomarkers emerged, such as those between Low Density Lipoproteins (LDL) and iron stores, possibly explaining perturbations in lipid metabolism in conditions of iron overload. However, causality between changes in biomarkers after dietary intervention and health improvement could not be established, except for a small subset of subjects with biomarkers 'out-of-clinically accepted range' at baseline.

Beside the presence of confounding factors and the difficulty to treat baseline variability, one limit of the above described approach could be the attempt of connecting the intervention diet and the biomarkers without considering the complexity of the food and of the foodhuman interactions.

\section{Challenges in food matrix description}

A high-resolution description of the food is the first step needed to unravel the complexity of the food-human interaction (Figure 1a). Foods are highly heterogeneous materials, and food components interactions are organized physically and chemically in the space along different length scales, thus generating a structural complexity in the food matrix. The effect of food structure on food disintegration and micronutrient release has been exemplary described in a recent work by Hiolle $e t$ al. [11 ]. The description of food structure usually relies on data gained by several imaging techniques, including Light Microscopy (LM), Scanning Electron Microscopy (SEM) [12], and Magnetic Resonance Imaging (MRI) [13]. Further details about the interactions between the food matrix and water, which is the diffusing medium for most nutrients, are also provided by nuclear magnetic relaxometry [14]. Image analysis and relaxometry allow to evaluate physicochemical and rheological features of the food, assessing their impact on bioaccessibility.

A different approach is given by modelling based on machine learning and data-driven techniques, which provides a fingerprint of the food matrix by merging its chemical and physical properties. Chemical fingerprints can be obtained through various techniques ranging from spectroscopy to gas chromatography. Accordingly, chemical descriptors can be concentrations and variations of concentration in time-resolved observations, proportional to spectral features, with the advantage of not needing to formally identify each single descriptor. If the quantification is robust, a correlation pattern of descriptors, even if unidentified, can be exploited along with other outputs for hypothesis-free fingerprinting.

Furthermore, the physical structure of a matrix can be described by merging quantitative measures of structural properties of the sample and multimodal imaging derived features. Techniques as multidimensional hyperspectral imaging analysis have proven to be effective for matrix characterization and oxidative damage detection [15] and to be suitable for descriptors extraction for fingerprinting. Magnetic Resonance Imaging can also give quantitative information about properties of the food matrix, such as tortuosity and porosity [16], enhancing the array of possible multimodal descriptors for machine learning and datadriven approaches.

Breaking down the challenges and the modelling aspects of food matrix effects on chemical reactivity, many levels of complexity are emerging [17]: i) effects on thermal stability of bioactive compounds and micronutrients; ii) thermodynamics and kinetics of reactions; iii) reactants concentration when catalytic phenomena are present; iv) diffusivity and partitioning of reactants among different phases of a matrix and v) enzymatic interactions. As a matter of fact, a chemical reaction occurring in food will yield a rate different from the rate obtained in ideal conditions (i.e. a very diluted solution) and varying from food matrix to food matrix. Such an effect can also account for a displacement of reaction equilibrium. Food matrix can thus change thermodynamic and kinetic properties of 
the reaction by acting on: i) concentrations of reactants and products, ii) activity coefficients, iii) diffusivity of reactants and products, as well as on iv) the temperature perceived by the reactants in each compartment of the system.

An exhaustive framework for the integration of fingerprinting and kinetics studies has been brought forth by Grauwet e al. [18]. Although focusing on the topic of evaluating the effects of extrinsic factors, such as processing on food quality changes, this work offered a comprehensive view on the techniques and approaches to be exploited for food characterization and extensive data generation (GC and HPLC MS, NMR based approaches). Moreover, the importance of linking fingerprinting with kinetics, through multi-response observation was highlighted. Multi-response observation for food means studying transformations in food. They do not occur isolated but, rather, within a network of reactions which are consequent to a variety of combinations of processing conditions. From a mathematical point of view, this is done by translating the reactions network into a system of coupled differential equations, using all the information extracted during studies aiming at characterizing the food matrix. The result is an insight into the rate constants of specific reactions steps, and their dependence on secondary variables (i.e. temperature, pressure, time, etc., in food processing), which refers to the study of a multiphasic reaction system shaping the food matrix. The paper by Grauwet et al. [18] also outlined some basic concepts behind multivariate data analysis (MVDA) techniques, which are crucial for information extraction in frameworks of the type proposed.

On the basis of the concept of multi-response kinetics, different compartments (i.e. the food, the GIT, the human metabolism) cannot be considered isolate systems. Therefore, data obtained in each compartment should be merged and integrated as part of a network of interactions. Modelling kinetics should consider complexity by building in-silico models including information from food matrix to the human body, including GIT.

\section{Challenges in the description of the impact of the food matrix on digestion}

The food matrix affects food components bioaccessibility/bioavailability influencing the entity and the kinetic of the release process in the GIT. Together with the individual 'intrinsic' variability (e.g. genetic polymorphisms) and the effect of the overall diet, the food matrix effect can lead to different digestion or absorption capacity of specific components, thus modulating the ultimate effect on physiology and health [19].

Research has focused on the development of standardized food models (SFM) for in-vitro experimental setups and investigations on three major levels for bioavailability modelling: bioaccessibility, absorption and transformation of nutrients within the GIT (Figure 1b). Mimicking the composition of representative diets allowed studying bioaccessibility of bioactive compounds. This aspect is affected by the heterogeneity of mixtures with different physical phases and nanostructures, in which nutrients tend to organize during digestion processes along the entire digestive apparatus. In a recent work by Zhang et al. [20], an SFM representing a typical US diet was proposed to investigate the effects of food matrices on bioaccessibility of nutraceutical and pesticides. Microstructures were characterized in each phase of the simulated in-vitro digestion using confocal fluorescence microscopy, also considering electrical properties. The work showed significant impact of the food matrix on bioaccessibility of bioactive compounds, and provided insights on the role of lipid digestion and its interaction with hydrophobic nutraceutical. Besides, it provided examples of possible important observables (i.e. physicochemical properties) derived from in-vitro set-ups. As examples, variations in electrical properties, particle size and microstructure distribution were acquired in each single stage of the digestion, to model different levels of complexity, as they impact on the interactions of enzymes with fat droplets. Indeed, the inclusion of these variables allowed to describe and explain the different ions release from food fragments and fat droplets in the different environments of the GIT.

Similar descriptors coming from in-vitro studies could play a crucial role in integrating the food matrix effect into reliable in-silico models considering the matrixdependent complexity of digestion kinetics. Of note, modelling structural interaction terms in kinetic equations systems, that is, the insertion of a quadratic damping term representing diffusion under certain conditions or a sigmoidal term representing percolation dynamics, could enhance in-silico simulations capabilities. When in-silico models must predict intertwined kinetics occurring in different compartments of the GIT, a set of observables as the one discussed above can be used to estimate and model interactions.

\section{Challenges in integrating food matrix description and metabolomics}

Observation derived from in-vitro experiments play a key role in the construction of appropriate kinetic descriptors of the food matrix effect on bioavailability. Since properties of the matrix influence the first phase of the food/ human interaction, that is, bioaccessibility, they influence all the subsequent phases. Therefore, a multi-compartmental modelling is needed (Figure 2) to account for complexity in an appropriate manner.

To build multi-compartmental models, patterns of blood and urine biomarkers can be adopted as proxies of the 
Compartments kinetics at different bioaccessibility parameters.

A simple model to visualize the effect of parametrizing bioaccessibility tied to food matrix. The simulations are run at identical starting concentration values and parameters of other compartments, except for bioaccessibility, whose parametrization is given by $\Lambda$. Differences in bioaccessibility propagates affecting the kinetics of a given observable in other compartments.
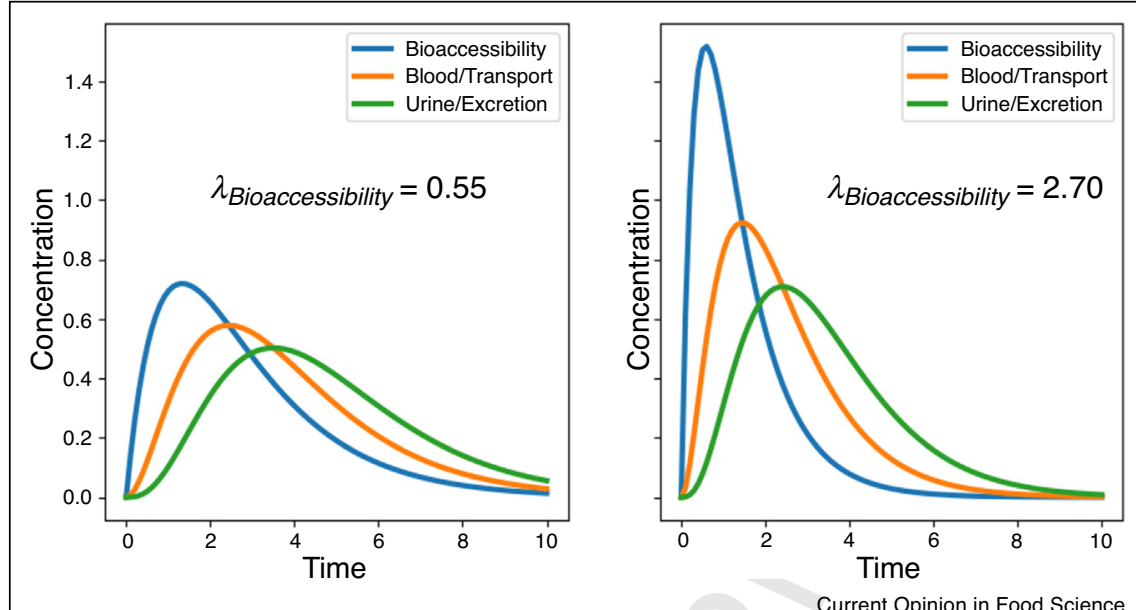

food/human interactions evolving during digestion and link them to the description of the food matrix.

Metabolomic of blood and urine is a key tool in the identification of dietary biomarkers that can be also used to classify and quantify food intake [8]. Many metabolomic studies are focused on expanding and validating Biomarkers of Food Intake (BFI). García-Pérez et al. [21] suggested an analytical pipeline based on correlation maps of $1 \mathrm{H}-\mathrm{NMR}$ identified metabolites for evaluation of dietary intake. That work evidenced tartaric acid as a dose responsive biomarker of grape intake, while proline betaine was indicated as a marker of citrus intake in the study by Gibbons et al. [22]. Clusters of biomarkers of milk, cheese and soy-based drink were identified by $\mathrm{M}$ ünger $e t$ al. with untargeted multiplatform analysis [23], and 3-methylhistidine was confirmed as specific for white-meat intake [24].

However, the ratio between validated and putative biomarkers of food intake is still very low. A guideline for evaluating the quality of candidate biomarkers was proposed by Dragsted et al. [25]. The adopted parameters included assessment of plausibility, dose response, time response, robustness, reliability, stability, analytical performance, and inter-laboratory reproducibility.

The most powerful perk of metabolomics is its ability to discover untargeted patterns of metabolites for subject classification. Single diet biomarkers might offer incomplete information and do not suffice when phenotyping free-living populations or trying to understand relationships between food consumption and disease risk
(Figure 1c). Garcia-Perez et al. [26 $6^{\circ}$ suggested the possibility to overcome biases related to self-reported dietary intake by a discrimination based on the fingerprinting of the whole urinary spectral profiles. Specific spectral archetypes were obtained from individuals kept in controlled feeding conditions and used for classification of dietary intake in free-living individuals. It was shown that the differentiation among dietary interventions was only allowed by whole patterns of urinary biomarkers embedded in the metabolic profile, while single specific biomarkers were not able to correctly classify the diet.

Considering whole patterns in place of single biomarkers can also mitigate the risk of misinterpreting metabolites concentration. As an example, urinary concentrations of TMAO can be associated with healthy, fish-rich diets; however, gut bacteria can synthesize TMAO from choline and hence high urinary and plasma concentrations can also originate from high red meat consumption, which is commonly tied to adverse health outcomes. Observation of the whole metabolome can disentangle such ambiguities.

The whole spectra of identified and unidentified signals, and the modification of their correlations, can allocate individuals in different metabotypes, thus enhancing baseline modelling and providing elements for intervention-related kinetics evaluation. One of the biggest challenges in this kind of approach is that compartmentalmodel computing needs a large amount of time-points data for robust parameter estimation. Many studies have thus focused on breaking down and simulating single compartment kinetics, focusing on absorption, digestion, 
transport or excretion. A recent work from Bjornson $e t$ al. $\left[27^{\circ}\right]$ highlighted the importance of evaluating interactions between absorption and transport phases. Using plasma samples, a novel non steady-state model was proposed, integrating metabolic characteristics of both apoB100 and apoB48 and the kinetics of triglycerides in response to a fat-rich meal. The model was proven to be physiologically relevant, providing information about apoB48 release in the basal and post-absorptive state, as well as about the contribution of intestine to Very LowDensity Lipoprotein (VLDL) pool size and kinetics. In a similar fashion, patterns of variation of spectral signals tied to metabolites may be used to intertwine multicompartmental kinetics, highlighting different profiles of response for different dietary interventions, while retaining inter-individual information and variability.

Usually, kinetic parameters can be drawn from at most two compartments (transport/absorption and excretion, if both serum and urine metabolomics are available) of the $\mathrm{N}$ possible macro-compartments of a model given by a chain of differential equations describing kinetics, such as the Bateman equations system. Fitting parameters for such equations becomes thus a challenge, especially when trying to model single-subject kinetics in the parameter space, unless time sampling is sufficiently high. Such a constraint should drive the experimental design of nutritional trials when kinetic information must be used for in-silico models. The high-resolution food description is also essential to conceive an informative quantification of bioaccessibility in the kinetic model. In Figure 2 the effect of including bioaccessibility in a simple multi-compartmental model is shown as a scalar parameter $(\kappa)$, to emphasize its effect propagating to every compartment. This quantification can be improved by finding functions of different parameters, extracted with the different techniques used to described food matrices in each experiment, and including them in the kinetic model.

\section{Conclusions}

The holistic approaches at the basis of foodomics are allowing the in-depth understanding of food matrix characteristics at molecular and supramolecular level. This is radically changing the nutritional approach that is now considering the food complexity as an important variable in the final effect of the diet.

Responses to food intake are not only specific for each individual but largely depend on the food matrix, including its modification due to processing. It is now clear that food cannot be considered a homogeneous mixture and it is time to give the right emphasis to the organization of the matrix.

The heterogeneous phases of the food matrix compartmentalize the biological systems and modulate the interactions among substrates and enzymes. This spatial restriction to the free diffusion of molecules may change during storage and/or processing of the food, which could be described as a dynamic system, and it is dramatically modified during digestion.

The destiny of a food component, from raw material to human compartments, is very complex. After digestion, accessible components are absorbed in a temporal and spatial distribution, some of them being meanwhile actively metabolized by the microflora. Active metabolization of absorbed components can occur already in the enterocyte before distribution to organs through the bloodstream. To predict it, in-vitro models simulating the physiological processes are adopted to the purpose of simplifying the interpretation of the results. However, these systems must undergo complex validation before being considered reliable predictors of in-vivo phenomena. This validation is enhanced by an in-silico step, that is, the construction of mathematical models and algorithms, which simplify the description of the different phases that food undergoes. These models are based on multi-factorial kinetic functions, whose parameters can be used to classify different categories of foods and of the corresponding individual responses.

To tackle the goal of these models, that is, the possibility to predict the impact of a food category on a class of individuals, and to overcome mathematical constrains on parameter estimation, huge amount of data from timeresolved studies are necessary.

The framework described herein considers many levels of complexity and highlights the importance of optimizing time-resolved experiments. This is a crucial step to implement robust algorithms and models based on machine learning and data-driven approaches, currently at the embryonic stage in this specific field of applications.

\section{Conflict of interest statement}

Nothing declared

\section{References and recommended reading}

Papers of particular interest, published within the period of review, have been highlighted as:

- of special interest

•• of outstanding interest

1. Betts JA, Gonzalez JT: Personalised nutrition: what makes you so special? Nutr Bull 2016, 41:353-359.

2. de Toro-Martín J, Arsenault BJ, Després JP: Precision nutrition: a review of personalized nutritional approaches for the prevention and management of metabolic syndrome. Nutrients 2017, 9 http://dx.doi.org/10.3390/nu9080913 pii: E913.

3. Laghi L, Picone G, Capozzi F: Nuclear magnetic resonance for foodomics beyond food analysis. TrAC Trends Anal Chem 2014 , 59:93-102.

4. Scalbert A, Brennan L, Manach C, Andres-Lacueva C, Dragsted LO, Draper J, Rappaport SM, van der Hooft J, 
Wishart DS: The food metabolome: a window over dietary exposure. Am J Clin Nutr 2014, 99:1286-1308.

5. Bordoni A, Capozzi F: Foodomics for healthy nutrition. Curr Opin Clin Nutr Metab Care 2014, 5:418-424.

6. Brodkorb André et al:: INFOGEST static in vitro simulation of gastrointestinal food digestion. Nat Protoc 2019, 14:991-1014

7. Dupont D, Le Feunteun S, Marze S, Souchon I: Structuring food to control its disintegration in the gastrointestinal tract and optimize nutrient bioavailability. Innov Food Sci Emerg Technol 2018, 46:83-4690.

8. Brouwer-Brolsma EM, Brennan L, Drevon CA, van Kranen $\mathrm{H}$ Manach C, Dragsted LO, Roche HM, Andres-Lacueva C Bakker SJL, Bouwman $\mathrm{J}$ et al.: Combining traditional dietary assessment methods with novel metabolomics techniques: present efforts by the food biomarker alliance. Proc Nutr Soc 2017, 76:619-627.

9. Tebani A, Bekri S: Paving the way to precision nutrition through metabolomics. Front Nutr 2019, 6:41.

10. Westerman K, Reaver A, Roy C, Ploch M, Sharoni E, Nogal B,

- Sinclair DA, Katz DL, Blumber JB, Blander G: Longitudinal analysis of biomarker data from a personalized nutrition platform in healthy subjects. Sci Rep 2018, 8:14685.

This work introduced a framework for nutritional recommendations based on custom biomarker correlation mapping. A set of common blood biomarkers of health were organized in a network of correlations as model to investigate pattern structures. Valuable information about average baseline functional complexity and subject-dependent variability has been found.

11. Hiolle M, Lechevalier V, Floury J, Boulier-Monthéan N, Prioul C,

- Dupont D, Nau F: In-vitro digestion of complex foods: how microstructure influences food disintegration and micronutrient bioaccessibility. Food Res Int 2020, 128

This paper highlights the impact of food structure on the digestion process, with different digestion pathways depending on the food matrix. It is concluded that the solubilization of macronutrients predominantly occurs at the gastric phase, while the hydrolysis occurs during the intestinal step.

12. El-Bakry Mamdouh, Sheehan Jeremiah: Analysing cheese microstructure: a review of recent developments. J Food Eng 2014, 125:84-96.

13. Groß D, Zick K, Guthausen G: Chapter four-recent MRI and diffusion studies of food structures. Annu Rep NMR Spectrosc 2017, 90:145-197.

14. Deng Ruoxuan, Janssen Anja EM, Vergeldt Frank J, Van As Henk, de Graaf Cees, Mars Monica, Smeets Paul AM: Exploring in-vitro gastric digestion of whey protein by time-domain nuclear magnetic resonance and magnetic resonance imaging. Food Hydrocolloids 2020, 99

15. Cheng W, Sun D-W, Pu H, Wei Q: Heterospectral twodimensional correlation analysis with near-infrared hyperspectral imaging for monitoring oxidative damage of pork myofibrils during frozen storage. Food Chem 2018, 248:119-127.

16. Schoeman L, Williams P, du Plessis A, Manley M: X-ray microcomputed tomography (mCT) for non-destructive characterization of food microstructure. Trends Food Sci Technol 2016, 47:10-24.
17. Capuano E, Oliviero T, van Boekel MAJS: Modeling food matrix effects on chemical reactivity: challenges and perspectives. Crit Rev Food Sci Nutr 2018, 58:2814-2828.

18. Grauwet Tara, Vervoort Liesbeth, Colle Ines, Van Loey Ann, Hendrickx Marc: From fingerprinting to kinetics in evaluating food quality changes. Trends Biotechnol 2014, 32:125-131.

19. Walther B, Lett A, Bordoni A, Tomás-Cobos L, Antonio Nieto J, Dupont D, Danesi F, Shahar D, Echaniz A, Re R et al.: GutSelf: inter-individual variability in the processing of dietary compounds by the human gastrointestinal tract. Mol Nutr Food Res 2019. in press.

20. Zhang Z, Zhang R, McClements DJ: Establishing the impact of food matrix effects on the bioaccessibility of nutraceuticals and pesticides using a standardized food model. Food Funct 2019, 10:1375-1385.

21. Garcia-Perez I, Posma JM, Chambers ES, Nicholson JK, Mathers JC, Beckmann M, Draper J, Holmes E, Frost G: An analytical pipeline for quantitative characterization of dietary intake: application to assess grape intake. J Agric Food Chem 2016, 64:2423-2431.

22. Gibbons $\mathrm{H}$, Michielsen CJR, Rundle M, Frost G, McNulty BA, Nugent AP, Walton J, Flynn A, Gibney MJ, Brennan L: Demonstration of the utility of biomarkers for dietary intake assessment; proline betaine as an example. Mol Nutr Food Res 2017, 61:1700037.

23. Münger LH, Trimigno A, Picone $G$, Freiburghaus $C$, Pimentel $G$ Burton KJ, Pralong FP, Vionnet N, Capozzi F, Badertscher R, Vergères $\mathrm{G}$ : Identification of urinary food intake biomarkers for milk, cheese, and soy-based drink by untargeted GC-MS and NMR in healthy humans. J Proteome Res 2017, 16:3321-3335.

24. Cheung W, Keski-Rahkonen $P$, Assi N, Ferrari $P$, Freisling $H$ Rinaldi S, Slimani N, Zamora-Ros R, Rundle M, Frost G et al.: A metabolomic study of biomarkers of meat and fish intake. Am J Clin Nutr 2017, 105:600-608.

25. Dragsted LO, Gao Q, Scalbert A, Vergères G, Kolehmainen, Manach C, Brennan L, Afman LA, Wishart DS, Andres Lacueva C et al:: Validation of biomarkers of food intake-critical assessment of candidate biomarkers. Genes Nutr 2018, 13:14.

26. Garcia-Perez I, Posma JM, Gibson R, Chambers ES, Hansen TH,

- Vestergaard H, Hansen T, Beckmann M, Pedersen O, Elliott P et al.: Objective assessment of dietary patterns by use of metabolic phenotyping: a randomised, controlled, crossover trial. Lancet Diabetes Endocrinol 2017, 5:184-195.

This research highlighted the capabilities of urine metabolic fingerprints obtained under controlled feeding conditions in phenotyping of free-living individuals. This approach is also useful in overcoming bias derived by self-reported dietary intake.

27. Björnson E, Packard CJ, Adiels M, Andersson L, Matikainen N,

- Söderlund S, Kahri J, Sihlbom C, Thorsell A, Zhou H et al: Investigation of human apoB48 metabolism using a new integrated non-steady-state model of apoB48 and apoB100 kinetics. J Intern Med 2019, 285:562-577.

The work provides an example on how to integrate complex kinetics between different compartments of the GIT. The model has proven to be physiologically relevant, giving insights about apoB48 release in the basa and postabsorptive state, as well as about the contribution of intestine to VLDL (very low-density lipids) pool size and kinetics. 